\title{
Low-loss germanium strip waveguides on silicon for the mid-infrared
}

\author{
Yu-Chi Chang, ${ }^{1, *}$ Vincent Paeder, ${ }^{1}$ Lubos Hvozdara, ${ }^{1}$ Jean-Michel Hartmann, ${ }^{2}$ and Hans Peter Herzig ${ }^{1}$ \\ ${ }^{1}$ Optics \& Photonics Technology Laboratory, Ecole Polytechnique Fédérale de Lausanne, Breguet 2, \\ 2000 Neuchâtel, Switzerland \\ ${ }^{2}$ CEA-LETI, Minatec Campus, 17 Rue des Martyrs, 38054 Grenoble, France \\ ${ }^{*}$ Corresponding author: yu-chi.chang@epfl.ch
}

Received May 1, 2012; accepted May 29, 2012;

posted June 4, 2012 (Doc. ID 167799); published July 11, 2012

\begin{abstract}
Mid-infrared photonics in silicon needs low-loss integrated waveguides. While monocrystalline germanium waveguides on silicon have been proposed, experimental realization has not been reported. Here we demonstrate a germanium strip waveguide on a silicon substrate. It is designed for single mode transmission of light in transverse magnetic (TM) polarization generated from quantum cascade lasers at a wavelength of $5.8 \mu \mathrm{m}$. The propagation losses were measured with the Fabry-Perot resonance method. The lowest achieved propagation loss is $2.5 \mathrm{~dB} / \mathrm{cm}$, while the bending loss is measured to be $0.12 \mathrm{~dB}$ for a $90^{\circ}$ bend with a radius of $115 \mu \mathrm{m}$. @ $2012 \mathrm{Optical}$ Society of America

OCIS codes: $230.7380,130.3060$
\end{abstract}

Since the advent of quantum cascade lasers (QCLs) [1] in 1994 and their spectroscopic applications [2], the technology of mid-infrared (mid-IR) laser spectroscopy is living a renaissance because of QCLs' high tunability, high power, and compact size. Most of the scientific effort is focused on active devices such as lasers [3] and detectors $[\underline{4}, \underline{5}]$, neglecting passive components such as lenses [ $[\underline{6}]$ or waveguides [7]. The integrated mid-IR spectroscopy needs elements such as waveguides, couplers, splitters, and resonators, which are the elementary structures to realize integrated optical devices.

Various materials have been used for mid-IR passive elements, such as silver halides, GaAs, and GaP [ 8 ]. Chalcogenide glasses were widely studied since the $1 \overline{9} 90 \mathrm{~s}$ [9]. Recent developments of silicon photonics based on silicon-on-insulator wafers or silicon-on-sapphire wafers are very promising because of their compatibility with silicon technologies, but their losses increase largely at longer wavelength due to the substrate leakage and the absorption in the buffer layers $[\underline{7}, \underline{10}]$.

Germanium has a broad transparent range in mid-IR and has been made into fibers [11] and planar waveguides on substrates like $\mathrm{ZnS}$ and IRTRAN2 $[\underline{12}, \underline{13}]$. It is nontoxic, and has the advantages of biocompatibility and silicon process compatibility. Low-loss monocrystalline germanium waveguides on silicon have been proposed [14] but not experimentally realized and evaluated in the mid-IR. Here we demonstrate a germanium strip waveguide on silicon and evaluated with a QCL at the wavelength of $5.8 \mu \mathrm{m}$.

As shown in Fig. 1, the waveguide is a strip structure consisting of $500 \mu \mathrm{m}$-long coupling elements, $350 \mu \mathrm{m}$-long funneling sections, and a several-millimeter straight section. The straight section is designed to be single mode in TM polarization (best adapted to the QCL) at a wavelength of $5.8 \mu \mathrm{m}$. It is $2.9 \mu \mathrm{m}$ wide and $2 \mu \mathrm{m}$ thick, and the effective refractive index is calculated to be 3.73 . Due to process variations, some waveguides are wider and may support an additional antisymmetric mode. However, the second mode was not observed during the measurements. A $90^{\circ}$ bend with a radius of $115 \mu \mathrm{m}$ is inserted into the waveguide in order to measure the sample away from the optical axis of the laser and avoid stray light. The bending loss is calculated to be in the range of $0.09 \mathrm{~dB}(2 \%)$.

The waveguide was fabricated with a $2 \mu \mathrm{m}$-thick monocrystalline germanium layer on a silicon substrate, which is covered with a $10 \mu \mathrm{m}$-thick intrinsic silicon buffer layer. The germanium layer was grown with reduced pressure chemical vapor deposition (RP-CVD). The strain in the germanium film is completely relaxed. Reference [15] presents details of the growth. Standard photolithography and fluorine chemistry based reactive ion etching were apllied to pattern the germanium layer. The inset of Fig. 1 shows the cross-section of the straight section of the waveguide. The edges of the substrate are then grinded and polished perpendicularly to the in-coupling and out-coupling parts of the waveguide. This allows direct access to the waveguide facets for optical measurements.

To measure the transmission loss, a method based on the Fabry-Pérot (F-P) fringe [16] is used. Unlike the cutback method, this method is nondestructive and insensitive to coupling variations. As shown in Fig. 2, the light is coupled into the waveguide using a $f / 1$ zinc selenide

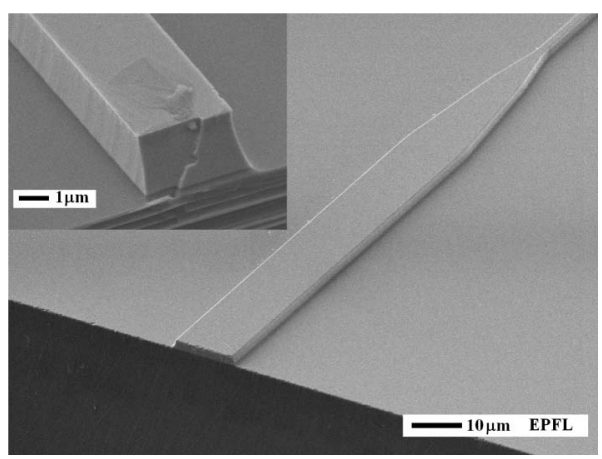

Fig. 1. Scanning electron microscopy image of the waveguide: the coupling part is $15 \mu \mathrm{m}$ wide, and the narrower part is $2.9 \mu \mathrm{m}$ wide. It is made from a $2 \mu \mathrm{m}$ thick monocrystalline germanium on silicon. The inset is the cross-section in the straight section. 


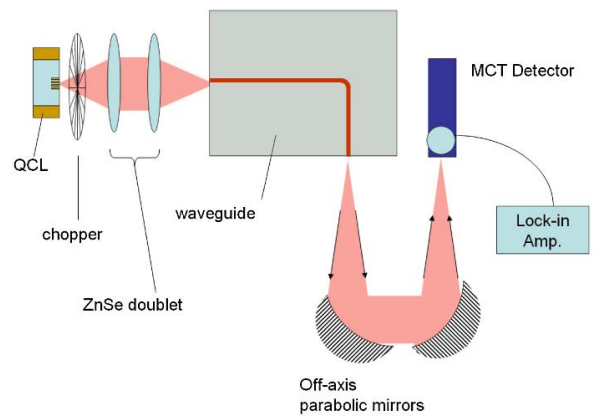

Fig. 2. (Color online) Experimental setup: light is coupled via a $f / 1$ doublet into the waveguide comprising a $90^{\circ}$ bend. It is collected via a pair of $f / 1$ off axis parabolic mirrors into an MCT detector.

doublet. After the bend, the transmitted light is coupled into a mercury-cadmium telluride (MCT) detector via a pair of $f / 1$ off-axis parabolic mirrors. The light source is a QCL at $5.8 \mu \mathrm{m}$ with the output power of $2 \mathrm{~mW}$. It is driven in a continuous wave operation mode, which gives a sharp and stable laser spectrum in order to resolve the F-P fringes of the resonance from the two facets of the waveguide. A chopper is added to the optical path to enable the signal evaluation using correlation techniques. The optical path-length is modulated by heating and cooling the sample from $30{ }^{\circ} \mathrm{C}$ to $50{ }^{\circ} \mathrm{C}$.

The propagation loss is evaluated from the contrast of the F-P fringes according to the formula

$$
\alpha L=\log \left(R \frac{1+\sqrt{P_{\min } / P_{\max }}}{1-\sqrt{P_{\min } / P_{\max }}}\right),
$$

where $P_{\min }$ is the measured output power in the minimum, $P_{\max }$ is the output power in the maximum, $L$ is the waveguide length, and $R$ is the reflectivity [17] of the waveguide facet calculated with the FDTD method. In our case, the reflectivity is 0.342 for a $15 \mu \mathrm{m}$-wide and $2 \mu \mathrm{m}$-thick germanium waveguide facet in air.

Figure 3 shows a typical trace of the measured F-P re-

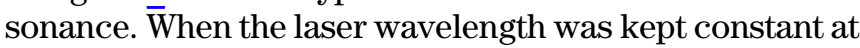
$5.8 \mu \mathrm{m}$, the transmitted light intensity was modulated by waveguide temperature changes. The decrease of the average value of the oscillations is attributed to system misalignment (because of different thermal expansion

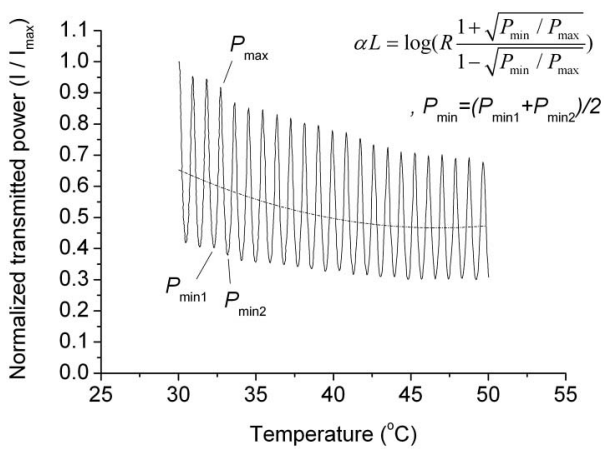

Fig. 3. Trace of the F-P resonance: the transmission intensity oscillates during temperature scanning. The fringe contrast $P_{\min } / P_{\max }$ of 0.42 corresponds to the loss of $2.5 \mathrm{~dB} / \mathrm{cm}$ for the waveguide length of $7.75 \mathrm{~mm}$.

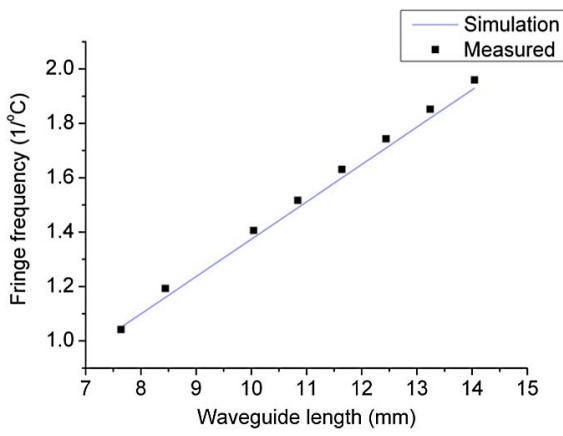

Fig. 4. (Color online) Oscillating frequencies of fringes from different waveguide lengths: longer waveguides yield higher oscillating frequencies during temperature scanning. The measurement result shows a good agreement with simulations.

of the elements). To minimize this effect on the loss calculation, we took the average of two adjacent minimums (maximums) for corresponding calculations of $P_{\min } / P_{\max }$.

For a $10 \mathrm{~mm}$-long waveguide, the total transmission is around $3 \%$, which is due to the high reflectivity at the waveguide facets and the coupling losses. The noise level was determined by laterally shifting the laser spot from the best alignment position until the signal reading was stable. The observed signal to noise ratio was about $30 \mathrm{~dB}$.

As the measured F-P fringes are based on the resonance of the cavity formed by the two facets of the waveguide, the length of the waveguide determines the free spectral range and also the oscillating frequency during temperature scanning. For a longer waveguide, the transmission intensity oscillated faster when temperature changed. The oscillating frequency can be simulated by taking into account the thermo-optic coefficient $d n / d T=0.000416^{\circ} \mathrm{C}-1$ and the thermal expansion $\alpha_{L}=6 \mathrm{E}-6{ }^{\circ} \mathrm{C}-1$ of germanium $[18,19]$. The calculated oscillating frequency, presented in Fig. 4 , is in good agreement with the measured results of waveguides with lengths from 7.7 to $14.2 \mathrm{~mm}$.

Measured with the F-P resonance method, the average propagation loss of eight different waveguides is about $3.5 \mathrm{~dB} / \mathrm{cm}$. This result is reconfirmed by measuring the transmitted power of these waveguides, which have different lengths, as shown in Fig. $\underline{5}$.

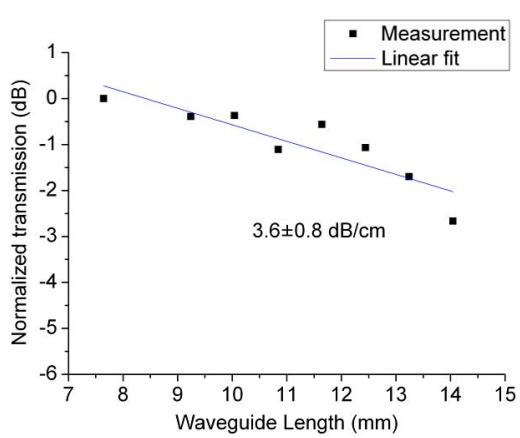

Fig. 5. (Color online) Transmission and waveguide lengths: the transmitted power is measured for eight waveguides with lengths from 7.7 to $14.2 \mathrm{~mm}$. The propagation loss of $3.6 \mathrm{~dB} / \mathrm{cm}$ reconfirmed the measurement result by the F-P resonance method. 


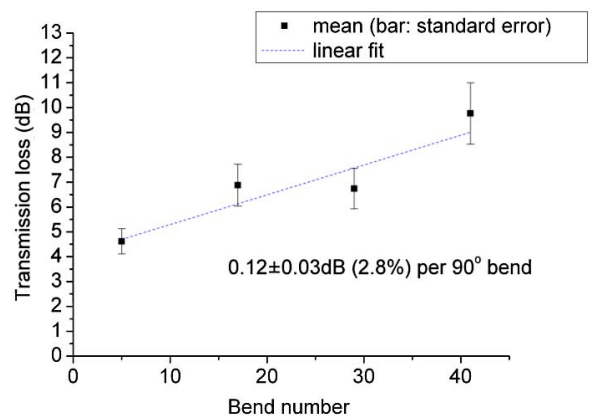

Fig. 6. (Color online) Bending loss measurement: keeping the waveguide length at $8.5 \mathrm{~mm}, 16$ waveguides with four different numbers of bends were measured. As the bend number increased, the transmission loss became higher by $0.12 \mathrm{~dB}$ (2.8\%) per $90^{\circ}$ bend, which has radius of $115 \mu \mathrm{m}$.

Since germanium has a low intrinsic absorption in the mid-IR range, the side-wall roughness is expected to partly contribute to the propagation loss. Besides, the relaxed germanium film contains misfit dislocations in the first few hundred nanometers from the silicongermanium interface. These defects may cause some loss.

The $90^{\circ}$ bend structure in each waveguide prevented undesired stray light from going into the detector but also introduced a bending loss for each measurement. This bending loss was measured with 16 waveguides, which have the same total length but four different bend numbers. As shown in Fig. 6 , a $90^{\circ}$ bend of the radius $115 \mu \mathrm{m}$ gave a loss of $0.12 \mathrm{~dB}(2.8 \%)$. When factoring in this bending loss, the lowest measured propagation loss is then $2.5 \mathrm{~dB} / \mathrm{cm}$ only, while the $P_{\min } / P_{\max }$ ratio is 0.42 for a $7.75 \mathrm{~mm}$-long waveguide.

This Letter demonstrates a low-loss germanium strip waveguide in the mid-IR wavelength range. It is single mode in TM polarization at the wavelength of $5.8 \mu \mathrm{m}$, and is realized with a $2 \mu \mathrm{m}$-thick monocrystalline germanium layer on a silicon substrate. It has a low propagation loss and a low bending loss.

This waveguide can be a building block for various integrated active and passive mid-IR photonics devices in a wide wavelength band, thanks to the low intrinsic absorption of germanium and silicon in this range. It is also an excellent platform for laser-spectroscopy, biomedical applications, and further development such as wavelength mixers and frequency combs. [르, 21$]$

This project is scientifically evaluated by the Swiss National Science Foundation (SNSF) and funded by the Swiss Federal program, Nano-Tera.

\section{References}

1. J. Faist, F. Capasso, D. L. Sivco, C. Sirtori, A. L. Hutchinson, and A. Y. Cho, Science 264, 553 (1994).

2. B. G. Lee, M. A. Belkin, R. Audet, J. MacArthur, L. Diehl, C. Pflügl, F. Capasso, D. C. Oakley, D. Chapman, A. Napoleone, D. Bour, S. Corzine, G. Höfler, and J. Faist, Appl. Phys. Lett. 91, 231101 (2007).

3. A. Evans, J. S. Yu, J. David, L. Doris, K. Mi, S. Slivken, and M. Razeghi, Appl. Phys. Lett. 84, 314 (2004).

4. D. Hofstetter, F. R. Giorgetta, E. Baumann, Q. Yang, C. Manz, and K. Köhler, Appl. Phys. Lett. 93, 221106 (2008).

5. D. Stanaszek, J. Piotrowski, A. Piotrowski, W. Gawron, Z. Orman, R. Paliwoda, M. Brudnowski, J. Pawluczyk, and M. Pedzinska, Proc. SPIE 7482, 4820M (2009).

6. R. Voelkel and M. Eisner, Proc. SPIE 4440, 40 (2001).

7. T. Baehr-Jones, A. Spott, R. Ilic, A. Spott, B. Penkov, W. Asher, and M. Hochberg, Opt. Express 18, 12127 (2010).

8. I. Paiss, F. Moser, and A. Katzir, Proc. SPIE 1420, 141 (1991).

9. X. Gai, T. Han, A. Prasad, S. Madden, D.-Y. Choi, R. Wang, D. Bulla, and B. Luther-Davies, Opt. Express 18, 26635 (2010).

10. G. Z. Mashanovich, M. M. Milošević, M. Nedeljkovic, N. Owens, B. Xiong, E. J. Teo, and Y. Hu, Opt. Express 19, 7112 (2011).

11. P. Mehta, M. Krishnamurthi, N. Healy, N. F. Baril, J. R. Sparks, P. J. A. Sazio, V. Gopalan, J. V. Badding, and A. C. Peacock, Appl. Phys. Lett. 97, 071117 (2010).

12. W. S. C. Chang and K. W. Loh, Appl. Opt. 10, 2361 (1971).

13. S. E. Plunkett, S. Propst, and M. S. Braiman, Appl. Opt. 36, 4055 (1997).

14. R. A. Soref, S. J. Emelett, and W. R. Buchwald, J. Opt. A 8, 840 (2006).

15. J. M. Hartmann, A. Abbadie, N. Cherkashin, H. Grampeix, and L. Clavelier, Semicond. Sci. Technol. 24, 055002 (2009).

16. G. Tittelbach, B. Richter, and W. Karthe, Pure Appl. Opt. 2, 683 (1993).

17. J. Buus, IEEE J. Quantum Electron. QE-17, 2256 (1981).

18. G. A. Slack and S. F. Bartram, J. Appl. Phys. 46, 89 (1975).

19. H. H. Li, J. Phys. Chem. Ref. Data 9, 561 (1980).

20. P. Del'Haye, A. Schliesser, O. Arcizet, T. Wilken, R. Holzwarth, and T. J. Kippenberg, Nature 450, 1214 (2007).

21. J. Liu, X. Sun, R. Camacho-Aguilera, L. C. Kimerling, and J. Michel, Opt. Lett. 35, 679 (2010). 